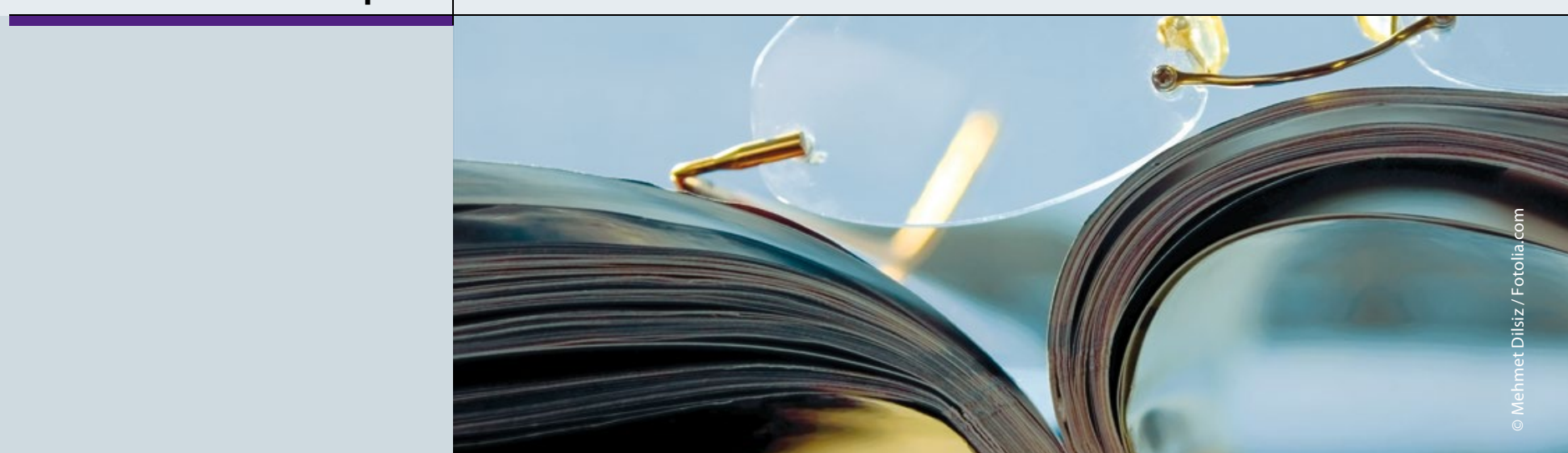

\section{Progesteron bei habituellen Aborten wirkungslos}

\section{Schwangere, die aus ungeklärten Gründen schon mehrere Fehlgeburten erlit- ten haben, haben einer neuen Studie zufolge keinen Nutzen von einer Pro- gesterontherapie im ersten Trimester. Die Chance auf eine Lebendgeburt wird dadurch nicht erhöht.}

$\mathrm{D}$ ie Bildung von Progesteron, zuerst im Corpus luteum, dann in der Plazenta, ist notwendig, um eine Schwangerschaft aufrechtzuerhalten. Theoretisch könnte das Gelbkörperhormon also bei Frauen mit rezidivierenden Aborten therapeutisch nutzbar sein. Das hatten auch die Ergebnisse eines Cochrane-Reviews von 2013 vermuten lassen: Eine Progesterontherapie im ersten Trimester war mit einem verminderten Fehlgeburtsrisiko assoziiert. Allerdings beruhte der Review auf nur vier kleinen Studien von minderer Qualität. Das könnte erklären, weshalb in einer neuen Studie mit dem verheißungsvollen Namen PROMISE die Ergebnisse nicht bestätigt werden konnten.

PROMISE (Progesterone in Recurrent Miscarriages) ist eine multizentrische, randomisierte, placebokontrollierte Doppelblindstudie mit 836 Frauen, die mindestens drei vorausgegangene Aborte unklarer Ursache hatten. Nach einer auf natürlichem Weg zustande gekommenen Schwangerschaft wendeten sie zweimal täglich entweder $400 \mathrm{mg}$ Progesteron oder Placebo in Form von Vaginalsuppositorien an. Die Therapie wurde möglichst bald nach einem positiven Schwangerschaftstest (Urintest), spätestens in der sechsten Gestationswoche begonnen und nach Woche zwölf abgesetzt.

Die Rate der Lebendgeburten nach der 24. Woche betrug mit dem Gestagen 65,8\% (262 von 398), unter Placebo 63,3\%
(271 von 428). Auch hinsichtlich der Dauer der Schwangerschaft, der Rate von Aborten und Totgeburten waren zwischen den Gruppen keine Unterschiede auszumachen. Ebenso war bei den lebend geborenen Kindern beider Gruppen das Gestationsalter gleich verteilt; vor der 34 . Woche waren $3,7 \%$ bzw. 3,8\% entbunden worden. Angeborene Fehlbildungen hatten 3,5\% bzw. 4,0\% der Kinder.

Die Autoren betonen, dass dieses negative Ergebnis mit einer Progesterondosis zustande gekommen sei, die „am oberen Ende des therapeutischen Fensters" liege. Die Behandlung bis zum Ende der zwölften Gestationswoche sei wahrscheinlich auch ausreichend lang gewesen, da sich die Aborte im Median schon nach weniger als acht Wochen ereignet hätten. Offen bleibt die Frage, ob eine Progesteronbehandlung schon in der Lutealphase vor Bestätigung der Schwangerschaft einen Nutzen hat.

Dr. Beate Schumacher

Coomarasamy A et al. Progesterone supplements do not improve outcome for recurrent miscarriages. NEJM 2015;373:2141-8

\section{Kommentar}

Die Wirkung von Progesteron oder 17-Hydroxiprogesteron zur Reduktion der Frühgeburtlichkeit sowie bei Zervixinsuffizienz und bei vorangegangenen Frühgeburten ist in gut randomisierten und kontrollierten Studien belegt und inzwischen leitlinienkonformer Standard in der Perinatologie. Der Effekt einer
Therapie mit dem schwangerschaftserhaltenden Hormon Progesteron bereits im ersten Trimenon bei Frauen mit rezidivierenden Aborten ist in einigen kleinen Studien untersucht worden und wird in der Cochrane-Database auch als effizient beschrieben.

In dieser jetzt im New England Journal of Medicine publizierten Studie wurde eine große Zahl von Frauen $(n=1.568)$ mit habituellen Aborten in der Vorgeschichte untersucht: 836 wurden innerhalb eines Jahres schwanger und kamen für die Studie infrage kamen, 404 Frauen wurden mit Progesteron behandelt, 432 Frauen erhielten Placebo. Als primärer Endpunkt wurde die Rate der Lebendgeburten nach der 24. Woche analysiert; diese war unter Gestagen-Behandlung und Placebo gleich hoch. Auch die Dauer der Schwangerschaft, die Rate von Aborten und Totgeburten war nicht unterschiedlich zwischen den Gruppen. Die Studie zeigt, dass eine Hype hinsichtlich eines Progesteron-Einsatzes bei allen Schwangerschaftsrisiken und Frauen mit negativem Schwangerschaftsausgang sicher nicht angezeigt ist. Es muss jedoch zwischen Fehl- und Frühgeburten, vor allen Dingen auch extremen Frühgeburten unterschieden werden. In der Studie wurde als primärer Endpunkt ja nur die Lebendgeburt nach der 24. Woche untersucht und das Gestationsalter der geborenen Kinder bei dieser oben erwähnten Indikation habitueller Aborte. Eine komplexe Pathophysiologie ist mit einem einzelnen Eingriff letztendlich nicht effektiv zu behandeln.

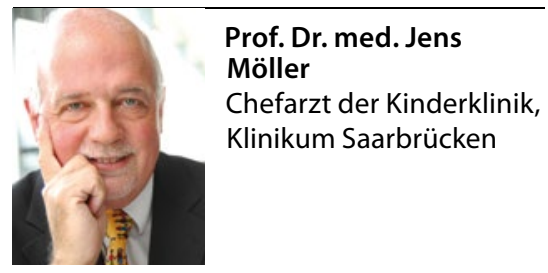

